УДК [024+025.5]:004

ББК 78.37c

DOI 10.20913/1815-3186-2019-2-19-25

\title{
ЦИФРОВИЗАЦИЯ ПРОЦЕССОВ ОБСЛУЖИВАНИЯ В БИБЛИОТЕКАХ - ЭТО УЖЕ РЕАЛЬНОСТЬ
}

\author{
(C) М. Ю. Нещерет, 2019
}

Российская государственная библиотека, Москва, Россия, e-mail: mпеr@rsl.ru

\begin{abstract}
Рассматриваются теоретические вопросы цифровизации в библиотеках, характеризуются современные формы и методы предоставления библиотечно-информационных услуг, способы коммуникации библиотек и пользователей. Раскрываются качественно новые возможности библиотечно-информационного обслуживания, обусловленные распространением цифровых технологий и робототехники. Примеры успешной реализации проектов цифровизации библиотечно-информационных услуг демонстрируют готовность библиотек к переходу на новый уровень развития. Автор приходит к выводу, что несмотря на то, что цифровизация существенно повышает оперативность предоставления услуг, положительно влияет на их качество и, следовательно, привлекает в библиотеки новых пользователей, библиотечно-информационное обслуживание включает также процессы, которые невозможны без участия человека, обладающего такими качествами, как способность к антиципации, эмоциональному переживанию, интуитивному прогнозированию. Необходимо осознавать и профессиональные потребности при использовании цифровых технологий в библиотечно-информационном обслуживании.
\end{abstract}

Ключевые слова: цифровизация, информатизация, библиотечно-информационное обслуживание, библиотечно-информационные услуги, библиотечно-информационные технологии

Для цитирования: Нещерет М. Ю. Цифровизация процессов обслуживания в библиотеках - это уже реальность // Библиосфера. 2019. № 2. С. 19-25. DOI: 10.20913/1815-3186-2019-2-19-25.

Digitization of user service processes in libraries is already a reality

M. Yu. Neshcheret

Russian State Library, Moscow, Russia; e-mail: mner@rsl.ru

The article considers theoretical issues of digitalization in libraries, characterizes modern forms and methods of providing library and information services, ways of communication between libraries and users. It reveals qualitatively new opportunities of library and information service due to spreading digital technologies and robotics in libraries. Cases of successful implementation of library and information services digitization projects demonstrate the library readiness to move to a new level of development. The author concludes that despite the fact that digitalization significantly increases services efficiency, effects positively on their quality and, consequently, attracts new users to libraries, library information services also include processes that are impossible without human participation with qualities such as the ability to anticipation, emotional experience, intuitive forecasting. Therefore, it is necessary to be aware of the boundaries and professional needs when using digital technologies in library and information services.

Keywords: digitalization, informatization, library and information services, library and information technologies

Citation: Neshcheret M. Yu. Digitization of user service processes in libraries is already a reality. Bibliosphere. 2019. № 2. P. 19-25. DOI: 10.20913/1815-3186-2019-2-19-25.

\section{Введение}

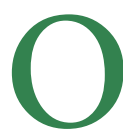

тличительной особенностью современного этапа развития общества является цифровизация. Под воздействием цифровых систем и технологий меняется окружающая нас реальность, цифровые средства коммуникации становятся неотъемлемой частью существования человека. Современными цифровыми сервисами пользуется практически каждый житель планеты, и материальный предметный мир дополняется виртуальным, легко трансформируемым миром объектов. Как глобальная тенденция мирового прогресса, цифровизация приобретает характерные черты мегатренда - крупномасштабного долгосрочного процесса, определяющего качественное содержание текущего этапа эволюции ${ }^{1}$.

1 Понятие «мегатренд» было предложено американским писателем и футурологом Джоном Нейсбитом в 1982 г. в книге «Мегатренды»; ее русский перевод вышел в 2003 г. (Москва : АСТ : Ермак, 2003. 380 с.). 
Под воздействием цифровых технологий происходит трансформация таких традиционных общественных институтов, какими являются библиотеки. Переосмысливаются принципы организации библиотечного пространства, методы библиотечно-информационной деятельности, ресурсного обеспечения библиотечных фондов, библиотеки стремятся использовать в своей каждодневной работе все возможности цифровизации. Цель данной статьи - показать, каким образом цифровизация проявляет себя в библиотечно-информационном обслуживании, как она становится повседневным атрибутом библиотечной практики. Примеры применения цифровых технологий в библиотеках позволяют продемонстрировать их положительную роль в модернизации этих учреждений культуры.

\section{1. Цифровизация - приоритетное направление культурной политики России}

В настоящее время цифровая трансформация в России существенно отстает от мировых уровней. Вклад цифрового сектора (производство и торговля информационно-технологическим оборудованием, сервисы и услуги, разработка программного обеспечения и цифровых товаров, телекоммуникации) в экономику России невелик в сравнении с развитыми странами Европы и Америки. Так, в 2017 г. его доля в экономике составила $3 \%$ ВВП, тогда как размер цифрового сектора во Франции $5,7 \%$, в Германии $-6,3 \%$, Великобритании $-7,1 \%$, США - 7,4\%, Швеции - 8,6\% [1, с. 2]. Вместе с тем Россия обладает потенциалом развития, о чем свидетельствуют темпы роста: с 2011 по 2015 г. цифровая экономика росла в 8,5 раза быстрее экономики России в целом; при этом наиболее значительный рост наблюдался в сфере государственных и муниципальных услуг [2, с. 8].

В 2017 г. Распоряжением Правительства Российской Федерации от 28 июля № 1632-р была утверждена национальная программа «Цифровая экономика Российской Федерации», направленная на создание необходимых условий для внедрения цифровизации во всех сферах социально-экономической деятельности. В феврале 2018 г. в Государственной Думе состоялись парламентские слушания на тему «Цифровизация в сфере культуры», на которых использование цифровых коммуникационных технологий в области осуществления всех видов культурной деятельности было объявлено приоритетной задачей государственной культурной политики. На слушаниях было отмечено, что для формирования информационного пространства знаний необходимо: создать безопасную информационную среду; усовершенствовать механизмы обмена знаниями; обеспечить функционирование Национальной электронной библиотеки (НЭБ) и иных государственных информационных систем, а также организовать доступ к ним максимально широкого круга пользователей; создать условия для популяризации русской культуры и науки за рубежом; принять меры по эффективному использованию современных информационных платформ для распространения достоверной и качественной информации; обеспечить насыщение рынка доступными и легальными медиапродуктами и сервисами российского производства [3].

\section{2. Чем отличается информатизация от цифровизации}

Массовое распространение термин «цифровизация» получил сравнительно недавно, и неудивительно, что его устоявшегося определения пока не существует. В широком смысле цифровизация - это «процесс внедрения цифровых технологий генерации, обработки, передачи, хранения и визуализации данных в различные сферы человеческой деятельности» [4, с. 17]. Ранее на протяжении нескольких десятилетий речь шла об информатизации преимущественно как о процессе внедрения методов информатики и информационных технологий в общественную жизнь.

Чем отличается информатизация от цифровизации? Информатизация - это совокупность электронных технологий, инструментарий в виде компьютеров, программ и информационных кабельных сетей, которые облегчают работу человека, но не заменяют его. Без информатизации не было бы и цифровизации, которая представляет собой более высокую, по сравнению с информатизацией, ступень технического развития общества и требует применения более сложных технологий. Главное отличие цифровизации от информатизации состоит в том, что она предполагает использование независимых цифровых систем с аналитическими и прогностическими функциями. Это еще не искусственный интеллект, но промежуточное звено между человеческим и искусственным разумом, так как в принятии решений определяющую роль по-прежнему играет человек - его интеллект, опыт, интуиция.

Следует также различать понятия «цифровизация» и «оцифровка». Оцифровка - это процесс преобразования аналоговой информации в цифровую, создание цифровых представлений существующих физических объектов или процессов. В библиотеках повсеместно осуществляется перевод документов на твердых носителях 
в электронную форму в целях сохранности оригиналов, раскрытия коллекций, повышения качества обслуживания пользователей. Уточняя содержание понятия «цифровизация», необходимо подчеркнуть, что речь идет не только о переводе информации в «цифру», но и о комплексном решении задач инфраструктурного, управленческого, поведенческого, культурного характера на базе специально разработанных технологических платформ [5, с. 17]. Их основными элементами являются облачные вычисления, интернет вещей, технологии больших данных и машинного обучения. Остановимся на характеристике этих элементов и рассмотрим их более подробно.

\section{3. Основные элементы цифровизации}

Облачные вычисления (cloud computing) - предоставление вычислительных ресурсов (серверов, хранилища, баз данных, сетевого оборудования, программного обеспечения, аналитики, интеллектуального анализа и т. д.) через интернет. Использование облачного сервиса освобождает от необходимости устанавливать обновления программного обеспечения, осуществлять проверку на вирусы и т.п. Обслуживание возлагается на провайдера сервиса, а за пользователем сохраняется минимальный набор необходимых функций.

Интернет вещей (Internet of things, IoT) - концепция вычислительной сети физических предметов («вещей»), оснащенных встроенными технологиями для взаимодействия друг с другом или с внешней средой с минимальным участием человека. По мнению экспертов, уже в 2009 г. количество вещей или материальных объектов, подключенных к интернету, превысило число людей, пользующихся Всемирной паутиной. Согласно прогнозам ученых, к 2020 г. к интернету будет подключено 50 млрд устройств [6]. Приведу примеры реально существующих интернет-вещей: устройство для поддержания комфортного уровня освещения и температуры в доме, которое автоматически регулирует положение жалюзи в зависимости от освещенности внутри помещения и вне его, а также от времени суток, погоды и пр.; «умный» термопластырь, который через настраиваемые промежутки времени определяет температуру тела и отправляет данные через приложение в смартфоне врачу; мусорный бак на солнечных батареях, который сам уплотняет мусор и сигналит дворникам, когда наполнится. Хорошо известная в России система «Яндекс.Навигатор» есть не что иное, как использование IоТ в управлении транспортом. Интернет вещей развивается не только в быту, но и на производстве. Например, в 2018 г. на выставке «Атомэкспо» были показаны «умные» каски, передающие информацию не только о том, надел ли сотрудник средство индивидуальной защиты, но и о том, где он находится. Они способны также информировать о сильных ударах по каске и о повышении температуры тела владельца, если он пришел на работу простуженным [7]

Большие данные (big data) - серия инструментов и методов обработки структурированных и неструктурированных данных значительных объемов и многообразия для получения воспринимаемых человеком результатов ${ }^{2}$. С данным термином связывают выражение «Volume, Velocity, Variety» - принципы, на которых строится работа с большими данными. Это объем информации, быстродействие ее обработки и разнообразие сведений, хранящихся в массиве. В последнее время к трем базовым принципам стали добавлять еще один - Value, что обозначает ценность информации в теоретическом или практическом плане, что оправдывало бы затраты на ее хранение и обработку. В частности, Министерство труда Германии сумело сократить расходы на 10 млрд евро, внедрив систему больших данных в работу по выдаче пособий по безработице. При этом было выявлено, что 1/5 часть граждан получает пособия необоснованно [8].

Машинное обучение (machine learning) - класс методов искусственного интеллекта, характерной чертой которых является не прямое решение задачи, а обучение в процессе применения решений множества сходных задач. Проще говоря, машинное обучение - процесс, в ходе которого компьютеры можно настраивать таким образом, чтобы они обучались сами.

\section{4. Цифровизация - модная тенденция или необходимость?}

Несмотря на то что цифровизация так явственно вторгается в нашу повседневную жизнь, вместе с ней приходят сомнения: цифровизация это модная тенденция или необходимость? Полагаю, не следует ждать, когда наступит полная определенность в понимании данного явления. Если исходить из того, что теория есть обобщение практики, то главная задача - не упустить возможности, которые открывают цифровые технологии.

Готовы ли учреждения культуры, в том числе библиотеки, встать на путь цифровизации?

2 Впервые термин «большие данные» (big data) появился в прессе в 2008 г., когда редактор журнала Nature Клиффорд Линч выпустил статью на тему развития технологий обработки большого количества данных. До 2009 г. термин рассматривался только с точки зрения научного анализа, но позже понятие big data стало использоваться в более широком значении. 
По всей вероятности, ответ на этот вопрос будет положительным. Первые шаги в направлении к цифровизации уже сделаны: в библиотеках используются информационные системы учета посетителей; осуществляется оцифровка культурного наследия; формируются электронные базы данных; создаются информационные порталы, виртуальные выставки; внедряются технологии дополненной (augmented reality) и виртуальной реальности (virtual reality) ${ }^{3}$. Цифровизация создает качественно новые возможности для библиотечно-информационного обслуживания с учетом того, что доступ к ресурсам и услугам библиотек сегодня осуществляется в режиме 24/7 из любой точки мира. Библиотеки становятся неотъемлемой частью не только отечественной, но и мировой информационной инфраструктуры [9].

Большое влияние на способы поиска и получения информации оказывают интернет-технологии, а электронные книги трансформируют привычные формы чтения. Уже существует НЭБ (https://нэб.рф), которая изначально задумывалась как точка доступа к научной и образовательной литературе. Ее целями являются формирование единого российского электронного пространства знаний и расширение доступности для граждан произведений научного и образовательного содержания.

\section{5. Как российские библиотеки адаптируются к цифровой реальности}

Во многих публичных библиотеках России действует онлайн-проект «ЛитРес: Библиотека» (biblio.litres.ru). Сервис позволяет, после авторизации на портале, получить бесплатный доступ к электронным книгам. Идея заимствована у существующего уже более 15 лет «Архива Интернета» и проекта «Открытая библиотека» (Open Library) (https://openlibrary.org). Основатель «Архива» Брюстер Кейл (Brewster Kahle) считает, что то, чего нет в интернете, для современного человека, по сути, просто не существует. Он призывает адаптировать библиотеки к новой цифровой реальности, сделав их фонды доступными для новых поколений читателей [10].

Процесс книговыдачи документов на бумажных носителях из библиотечного фонда значительно упростило внедрение технологий радиочастотной идентификации (radio frequency

3 Дополненная реальность - это технология добавления, внедрения в реальную жизнь, в трехмерное поле восприятия человека виртуальной информации, которая воспринимается как элементы реальной жизни. Виртуальная реальность - искусственно создаваемый смоделированный компьютерный мир, в который погружается человек. Однако, попадая в виртуальный мир, мы осознаем, что находимся в искусственно созданном пространстве. identification, RFID). RFID-метка - это наклейка небольшого размера с уникальным кодом, по которому информационная система отличает один объект от другого. С помощью RFIDсчитывателя идентификационные данные считываются и передаются через соответствующие каналы связи специальной программе. Программное обеспечение использует уникальную информацию для идентификации объекта, к которому поднесено считывающее устройство. RFID-системы приема/выдачи книг избавляют сотрудников библиотеки от рутинных операций, связанных с заполнением бланков и поиском изданий. Читатели самостоятельно оформляют книговыдачу, записывая ее на электронный формуляр с помощью терминала самообслуживания. Во время книговыдачи осуществляется деактивация RFID-метки, и издание переходит в статус «выдано». Читатель также может проверить состояние собственного формуляра и узнать о задолженности и сроках возврата числящихся за ним изданий. Вернуть книги можно с помощью модульной системы возврата книг. При возврате издания сканируются, на экране появляется информация о том, что книги списаны с электронного формуляра пользователя. Затем издания отправляются на транспортерную ленту станции сортировки и попадают в контейнеры, которые доставляют их к месту хранения. Развитие систем электронного заказа и электронной книговыдачи - примеры цифровизации библиотечных услуг на основе принципов доступности, оперативности, экономичности и самообслуживания.

\section{6. Цифровизация в зарубежных библиотеках}

Быстрыми темпами идет процесс цифровизации в зарубежных библиотеках. Во многих университетских библиотеках США - Библиотеке им. Джеймса Ханта-младшего (James B. Hunt Jr. Library) Университета Северной Каролины (North Carolina State University) и др. - действует система компактного хранения и автоматической выдачи книг BookBot, рассчитанная на 2-3 млн изданий. Книги хранятся в 15-метровых стальных боксах. Каждая книга имеет свой оригинальный штрихкод, позволяющий искать ее при помощи виртуального браузера. Робототехнический механизм соединен с электронным каталогом и системой электронного заказа: как только книга запрашивается пользователем, система автоматически посылает соответствующую команду для обнаружения ее местонахождения. Через несколько минут библиотекарь извлекает из ячейки запрошенную книгу, чтобы доставить ее пользователю [11].

В декабре 2018 г. в Хельсинки (Финляндия) открылось новое здание библиотеки Oodi, 
в которой работает автоматическая система возврата книг. В процессе задействованы два робота-манипулятора и три мобильных робота. Книги из рук читателя попадают на сортировку по 15 тематическим разделам, роботы сами раскладывают их по ящикам, вызывают лифт и поднимают ящики на третий этаж, в книгохранилище [12].

В Калифорнии, в Кремниевой долине, робот BookBot обходит дома, собирая книги, чтобы вернуть их в библиотеку. Прежде чем явиться с визитом, робот самостоятельно обзванивает пользователей. В первые месяцы робота сопровождал библиотекарь-куратор, чтобы убедиться, что он работает правильно [13].

В Публичной библиотеке Китченера (Kitchener Public Library) (Онтарио, Канада) читатели для поиска книги пользуются приложением librARi (https://youtu.be/aMWDnvRBKiw). Оно помогает обнаружить издание при наведении камеры на стеллажи или предлагает дополнительную литературу.

Об актуальности использования робототехники в библиотеках свидетельствует следующий факт: Секция информационных технологий Международной федерации библиотечных ассоциаций и учреждений (ИФЛА) совместно с Техническим университетом прикладных наук Вильдау (Technical University of Applied Sciences Wildau) (Бранденбург, Германия) в 2019 г. плани рует провести конференцию на тему «Роботы в библиотеках: вызов или возможность» ("Robots in Libraries: Challenge or Opportunity?”), на которой будут рассматриваться перспективы использования робототехнических технологий в библиотеке (https://2019.ifla.org/cfp-calls/ information-technology-section).

Необходимо заметить, что первые роботы в библиотеках появились уже достаточно давно. В 2003 г. в прессе было опубликовано сообщение о том, что в Японии создан «электронный библиотекарь», который позволяет читать книги в библиотеке, находясь вне ее - в офисе или дома. Для передвижения между книжными полками и объезда препятствий робот использует лазерную систему. Машина способна выбрать книгу и перелистывать страницы, передавая содержание на дисплей пользователя [14]. В 2004 г. сотрудники Университета Хайме I (Universidad Jaime I) (г. Кастельон, Испания) приступили к созданию библиотечного робота. По мнению разработчиков, библиотека - наиболее подходящее место для внедрения робототехники, так как здесь служебные роботы находятся под контролем библиотекарей. Робота можно попросить принести книгу с полки. Программа распознавания речи сопоставляет ее название с кодом классификации, хранящимся в базе данных. Местонахождение книги робот обнаруживает при помощи встроенной системы навигации с инфракрасными и лазерными датчиками. Он может прочитать названия книг, используя средства распознавания изображений и печатных знаков [15].

Сегодня роботы-библиотекари «трудятся» во многих библиотеках - как за рубежом, так и в России. В 2015 г. презентация робота Элби (сокращение от «электронный библиотекарь») состоялась в Центральной детской библиотеке Сургута. Его технические возможности позволяют побывать в библиотеке даже тем детям, которые по какой-либо причине не могут посетить ее самостоятельно. Зная логин и пароль нового библиотекаря, ребенок совершит виртуальное путешествие по библиотеке, сможет посетить мастер-класс, посмотреть спектакль кукольного театра, выбрать книгу для чтения. Элби водит экскурсии по библиотеке, читает вместе с детьми книги и отвечает на вопросы посетителей [16].

В некоторых библиотеках роботы читают книги вслух с целью приобщения к чтению маленьких пользователей, но такое применение новых технологий вызывает сомнения: едва ли роботы способны заменить живое общение и эмоциональное участие, которое исходит от взрослых, читающих книги детям.

Еще раньше в библиотеках, преимущественно университетских, появились компьютерные программы для общения с пользователями. Чатботы выполняют справочные функции: отвечают на вопросы по заранее подготовленным вариантам (скриптам). Они не могут вести свободный диалог, но разработка ботов, понимающих естественный язык, уже ведется.

Процесс цифровизации существенно изменил технологию избирательного распространения информации (ИРИ). Данная услуга в течение длительного времени осуществлялась библиотеками преимущественно в ручном или полуавтоматизированном режиме. С середины 1990-х гг. за рубежом развивается ИРИ-сервис (Selective dissemination of information, Current awareness services, Alerting services и пр.), основанный на интернет-технологиях. В нашей стране ИРИ-онлайн также становится востребованной услугой, преимущества которой очевидны: она обеспечивает непрерывность в информировании пользователей о выходе актуальных публикаций, способствует повышению целенаправленности научной информации, экономит время ученых и специалистов при поиске релевантной информации. Сейчас в мире существует множество вариантов ИРИ, которые отличаются друг от друга принципами построения, формами предоставления информации и составом документных ресурсов. Функциями ИРИ оснащены сайты библиотек, издательств (Elsevier, Springer, Wiley и др.), компании, формирующие 
базы научной информации Web of Science, Scopus, Current Contents, ProQuest и др. Пользователь самостоятельно регистрируется в избранной им системе ИРИ, создает индивидуальный тематический профиль, вводит ключевые слова по теме своих научных интересов, выбирает ресурсы, устанавливает частоту и формат оповещений. Пакетные сообщения система ИРИ формирует автоматически.

\section{Выводы}

Итак, мы являемся очевидцами формирования принципиально иного типа библиотечно-информационного обслуживания. С распространением цифровых технологий происходят качественные изменения форм, методов библиотечно-информационных услуг, способов коммуникации между библиотекарем и пользователем. Все более востребованными становятся не столько информационные ресурсы, сколько системы управления ими. Программы интеллектуального поиска позволяют быстро находить нужную информацию и доставлять ее пользователю. Цифровизация существенно

\section{Список источников}

1. Титов Б. Россия: от цифровизации к цифровой экономике : исследование. URL: http://stolypin. institute/wp-content/uploads/2018/09/issledovanie tsifrovaya-ekonomika-14-09-18-1.pdf (дата обращения: 08.02.2019).

2. Манахова И. В. Цифровое будущее и глобальная экономическая безопасность // Экономическая безопасность и качество. 2018. № 1. С. 6-11.

3. Цифровизация в сфере культуры. Законодательство и правоприменительная практика. URL: http://www.unkniga.ru/news/8004 (дата обращения: 01.02.2019).

4. Индустрия российских медиа: цифровое будущее : акад. моногр. / Е. Л. Вартанова, А.В. Вырковский, М.И. Максеенко, С. С. Смирнов. Москва : МедиаМир, 2017. 160 с.

5. Плотников В. А. Цифровизация производства: теоретическая сущность и перспективы развития в российской экономике // Известия Санкт-Петербургского государственного экономического университета. 2018. № 4. С. 16-24.

6. Дейв Э. Интернет вещей: как изменится вся наша жизнь на очередном витке развития Всемирной сети. URL: https://www.cisco.com/c/ $\mathrm{dam} / \mathrm{global} / \mathrm{ru}$ _ru/assets/executives/pdf/internet_ of_things_iot_ibsg_0411final.pdf (дата обращения: 08.02.2019).

7. Волошина В. Революция в мозгах, или чем цифровизация отличается от автоматизации // повышает оперативность предоставления услуг, положительно влияет на их качество и, следовательно, привлекает в библиотеки новых пользователей.

Вместе с тем необходимо помнить, что библиотечно-информационное обслуживание, помимо алгоритмизируемых операций, включает процессы, которые невозможны без участия человека, обладающего такими качествами, как способность к антиципации, эмоциональному переживанию, интуитивному прогнозированию. Так, эмоциональное переживание способствует успешному осуществлению библиографического поиска: в зависимости от совокупности имеющихся психологических установок осуществляется избирательный отбор релевантной информации.

Используя цифровые технологии, необходимо ориентироваться на профессиональные потребности и в то же время осознавать границы, за которыми применение цифровых систем не только становится нерациональным, но и может отрицательно повлиять на качество библиотечно-информационного обслуживания. Любое цифровое переосмысление должно учитывать ценность, которую библиотека предоставит пользователям.

Управление производством. 2018. № 6. URL: http:// www.up-pro.ru/library/strategy/tendencii/cyfraavtomat.html (дата обращения: 08.02.2019).

8. Индустрия 4.0: Big Data. URL: www.rce.dasi27. ru/news/industriia-40-big-data (дата обращения: 01.02.2019).

9. Шрайберг Я. Л. Формирование единого пространства знаний на базе сетевой информационной инфраструктуры в условиях становления и развития современной цифровой экономики : ежегод. докл. Четвертого междунар. проф. форума «Крым-2018» // Научные и технические библиотеки. 2018. № 9. С. 3-75.

10. Brewster $K$. Transforming our libraries into digital libraries: a digital book for every physical book in our libraries. URL: https://archive.org/details/ TransformingourLibrariesintoDigitalLibraries 102016 (дата обращения: 22.02.2019).

11. Payne L. Winning the space race: expanding collections and services with shared depositories // American Libraries. 2014. URL: https:// americanlibrariesmagazine.org/2014/09/23/winningthe-space-race/ (дата обращения: 22.02.2019).

12. «Ода» всем: в Хельсинки открылась библиотека с роботами и лучшим видом на город // Фонтанка.ру : [сайт] РВС. 2018. 8 дек. URL: https://www.fontanka.ru/2018/12/07/140 (дата обращения: 03.02.2019).

13. Baldassari E. Meet BookBot: mountain view library's newest robot helper only in Silicon Valley does a robot return your library books. URL: https:// 
www.siliconvalley.com/2019/03/09/meet-bookbotmountain-view-librarys-newest-robot-helper/.

14. Робот-библиотекарь способен передавать содержание книги через Интернет // Деловая пресса. 2003. № 49. URL: http://businesspress.ru/ newspaper/article_mId_37_aId_286371.html (дата обращения: 03.02.2019).

15. Твист Дж. Библиотечных червей заменят роботы // BBCRussian. 2004. 21 июля. URL: http://news.bbc.co.uk/hi/russian/sci/tech/ newsid_3914000/3914065.stm (дата обращения: 23.02.2019).

16. Жаткина Л., Латыпов Р. Этот электронный библиотекарь должен привлечь в библиотеки современных детей. URL: https://ugra-tv.ru/ news/society/etot_elektronnyy_bibliotekar_dolzhen_ privlech_v_biblioteki_sovremennykh_detey (дата обращения: 23.02.2019).

\section{References}

1. Titov B. Rossiya: ot tsifrovizatsii $k$ tsifrovoi ekonomike [Russia:from digitalization to digitaleconomy].URL: http://stolypin.institute/wp-content/uploads/2018/09/ issledovanie_tsifrovaya-ekonomika-14-09-18-1.pdf (accessed 08.02.2019). (In Russ.).

2. Manakhova I.V. Digital future and global economic security. Ekonomicheskaya bezopasnost' $i$ kachestvo, 2018, 30 (1), 6-11. (In Russ.).

3. Tsifrovizatsiya $v$ sfere kul'tury. Zakonodatel'stvo i pravoprimenitelnaya praktika [Digitization in the field of culture. Legislation and law enforcement practice]. URL: http://www.unkniga.ru/news/8004 (accessed 01.02.2019). (In Russ.).

4. Vartanova E. L., Vyrkovskii A.V., Makseenko M. I., Smirnov S.S. Industriya rossijskih media: tsifrovoe budushchee [Russian media industry: a digital future]. Moscow, MediaMir, 2017. 160 p. (In Russ.).

5. Plotnikov V.A. Digitalization of production: theoretical nature and development prospects in the Russian economy. Izvestiya Sankt-Peterburgskogo gosudarstvennogo ekonomicheskogo universiteta, 2018, 112 (4), 16-24. (In Russ.).

6. Deiv E. Internet veshchei: kak izmenitsya vsya nasha zhizn' na ocherednom vitke razvitiya Vsemirnoi seti [Internet of things: how our whole life will change at the next stage of the World Wide Web development]. URL: https://www.cisco.com/c/dam/global/ ru_ru/assets/executives/pdf/internet_of_things_iot_ ibsg_0411final.pdf (accessed 08.02.2019). (In Russ.).
7. Voloshina V. The revolution in brains, or how digitalization and automation differ. Upravlenie proizvodstvom, 2018, 6. URL: http://www.up-pro.ru/ library/strategy/tendencii/cyfra-avtomat.html (accessed 08.02.2019). (In Russ.).

8. Industriya 4.0: Big Data. URL: www.rce.dasi27. $\mathrm{ru} /$ news/industriia-40-big-data (accessed 01.02.2019). (In Russ.).

9. Shraiberg Ya.L. Forming a common knowledge space based on the network information infrastructure under conditions of modern digital economy formation and development : annu. rep. of the Fourth Intern. prof. forum "Crimea-2018”. Nauchnye i tekhnicheskie biblioteki, 2018, 9, 3-75. (In Russ.).

10. Brewster K. Transforming our libraries into digital libraries: a digital book for every physical book in our libraries. URL: https://archive.org/details/ TransformingourLibrariesintoDigitalLibraries102016 (accessed 22.02.2019).

11. Payne L. Winning the space race: expanding collections and services with shared depositories. American Libraries, 2014. URL: https://americanlibrariesmagazine.org/2014/09/23/winning-the-space-race/ (accessed 22.02.2019).

12. "Ode" to everyone: a library with robots and the best view of the city opened in Helsinki. Fontanka.ru. 2018. URL: https://www.fontanka.ru/2018/12/07/140 (accessed 03.02.2019). (In Russ.).

13. Baldassari E. Meet BookBot: mountain view library's newest robot helper only in Silicon Valley does a robot return your library books. URL: https://www.siliconvalley.com/2019/03/09/ meet-bookbot-mountain-view-librarys-newest-robot-helper/(accessed 22.02.2019).

14. A librarian robot is able to transmit book content over the Internet. Delovaya pressa, 2003, 49. URL: http://businesspress.ru/newspaper/article_mId_37_ aId_286371.html (accessed 03.02.2019). (In Russ.).

15. Tvist J. Library worms will be replaced by robots. BBCRussian. 2004, 21 July. URL: http://news.bbc. co.uk/hi/russian/sci/tech/newsid_3914000/3914065. stm (accessed 23.02.2019). (In Russ.).

16. Zhatkina L., Latypov R. Etot elektronnyi bibliotekar' dolzhen privlech' $v$ biblioteki sovremennyhk detei [This electronic librarian should attract nowadays children to libraries.]. URL: https://ugra-tv.ru/ news/society/etot_elektronnyy_bibliotekar_dolzhen_ privlech_v_biblioteki_sovremennykh_detey (accessed 23.02.2019). (In Russ.).

Материал поступил в редакцию 09.03.2019

Сведения об авторе: Нещерет Марина Юрьевна - кандидат педагогических наук, ведущий научный сотрудник РГБ, ORCID: 0000-0001-7155-2097 